\title{
BIBO STABILITY OF DISCRETE CONTROL SYSTEMS WITH SEVERAL TIME DELAYS
}

\author{
ESSAM AWWAD, ISTVÁN GYŐRI, AND FERENC HARTUNG
}

Received 23 May, 2017

\begin{abstract}
This paper investigates the bounded input bounded output (BIBO) stability in a class of control system of nonlinear difference equations with several time delays. The proofs are based on our studies on the boundedness of the solutions of a general class of nonlinear Volterra difference equations with delays.
\end{abstract}

2010 Mathematics Subject Classification: 39A30; 93C55

Keywords: boundedness, Volterra difference equations, bounded input bounded output (BIBO) stability, difference equations with delays

\section{INTRODUCTION}

Time delays play an important role in control systems, since a delay naturally appears when a system wants to measure or react to information. Stability or stabilization of a system is one of the central question which is investigated in control theory [10-12]. Because of its simplicity, the bounded input bounded output (BIBO) stability of control systems is widely investigated. The sufficient conditions for BIBO stability of control systems without delays are obtained in $[18,19]$ by using Liapunov function techniques. More recently many researchers have focused their interest on the BIBO stability of nonlinear discrete and continuous feedback control systems with or without delays $[1,2,5-9,13-15,17]$.

In this paper we consider a class of discrete control systems with multiple time delays. We search for delayed feedback controls such that the corresponding closed loop system be BIBO stable. We rewrite the closed loop system as an equivalent nonlinear Volterra difference equation (VDE) with delays. The BIBO stability results are based on our theorem which formulate sufficient conditions for the boundedness of the solutions of delayed VDEs. The results presented in this manuscript extend the methods introduced in [1] for nonlinear differential equations with a single delay and boundedness of ordinary VDEs presented in [3].

The structure of the manuscript is the following. Section 2 contains the precise problem statement, the definitions of BIBO stability and local BIBO stability, and we 
rewrite our closed loop control equation as an equivalent VDE. Section 3 formulates sufficient conditions for the boundedness of a general class of nonlinear VDEs with multiple delays. Section 4 contains our BIBO stability results for cases when the nonlinearity has a sub-linear, linear or super-linar estimates.

In the rest of this section we introduce some notations which will be used throughout this paper. $\mathbb{R}, \mathbb{R}_{+}, \mathbb{R}^{d}$ and $\mathbb{R}^{d \times d}$ denote the set of real numbers, nonnegative real numbers, $d$-dimensional real column vectors and $d \times d$-dimensional real matrices, respectively. The maximum norm on $\mathbb{R}^{d}$ is denoted by $\|\cdot\|$, i.e., $\|x\|:=$ $\max _{1 \leq i \leq d}\left|x_{i}\right|$, where $x=\left(x_{1}, \ldots, x_{d}\right)^{T}$. The matrix norm on $\mathbb{R}^{d \times d}$ generated by the maximum vector norm will be denoted by $\|\cdot\|$, as well. Let $\mathbb{Z}_{+}$and $\mathbb{N}$ be the set of nonnegative and positive integers, respectively. $L^{\infty}\left(\mathbb{Z}_{+}, \mathbb{R}^{d}\right)$ will denote the set of bounded sequences $r: \mathbb{Z}_{+} \rightarrow \mathbb{R}^{d}$ with norm $\|r\|_{\infty}:=\sup _{n \in \mathbb{Z}_{+}}\|r(n)\|$. Let $\tau>0$ be a fixed integer, $S\left([-\tau, 0], \mathbb{R}^{d}\right)$ denotes the set of finite sequences

$$
\left\{\psi:\{-\tau,-\tau+1, \ldots, 0\} \rightarrow \mathbb{R}^{d}\right\}
$$

and $\|\psi\|_{\tau}:=\max _{-\tau \leq n \leq 0}\|\psi(n)\|$. For a given sequence $x$ and an integer $n$ the forward difference operator is defined by $\Delta x(n):=x(n+1)-x(n)$.

\section{Problem statement}

In this paper we consider the nonlinear discrete control system with several delays

$$
\begin{aligned}
\Delta x(n) & =g\left(n, x\left(n-\sigma_{1}(n)\right), \ldots, x\left(n-\sigma_{\ell}(n)\right)\right)+u(n), \quad n \in \mathbb{Z}_{+}, \\
y(n) & =C x(n), \quad n \in \mathbb{Z}_{+} .
\end{aligned}
$$

Here $x(n) \in \mathbb{R}^{d}$ is the state vector, $u(n) \in \mathbb{R}^{d}$ is the input vector and $y(n) \in \mathbb{R}^{d_{1}}$ is the output vector of the system (2.1), $C \in \mathbb{R}^{d_{1} \times d}$ is a constant matrix, $\sigma_{i}: \mathbb{Z}_{+} \rightarrow$ $\mathbb{Z}_{+}, i=1, \ldots, \ell$ are bounded delay functions, and the nonlinear function $g: \mathbb{Z}_{+} \times$ $\underbrace{\mathbb{R}^{d} \times \ldots \times \mathbb{R}^{d}}_{\ell} \rightarrow \mathbb{R}^{d}$ satisfies

$$
\left\|g\left(n, x^{(1)}, \ldots, x^{(\ell)}\right)\right\| \leq b(n) \varphi\left(\max _{1 \leq m \leq \ell}\left\|x^{(m)}\right\|\right), \quad n \in \mathbb{Z}_{+}, x^{(1)}, \ldots, x^{(\ell)} \in \mathbb{R}^{d},
$$

where $b(n)>0$ for all $n \in \mathbb{Z}_{+}$, and $\varphi: \mathbb{R}_{+} \rightarrow \mathbb{R}_{+}$is a monotone nondecreasing mapping.

Our general problem (2.1) satisfying condition (2.2) includes, e.g., linear control systems

$$
\Delta x(n)=A_{1}(n) x\left(n-\sigma_{1}(n)\right)+\cdots+A_{\ell}(n) x\left(n-\sigma_{\ell}(n)\right)+u(n), \quad n \in \mathbb{Z}_{+},
$$


and nonlinear control systems of the form

$$
\Delta x_{i}(n)=\sum_{j=1}^{d} a_{i j}(n) x_{j}^{p}\left(n-\sigma_{j}(n)\right)+u_{i}(n), \quad n \in \mathbb{Z}_{+}, i=1, \ldots, d,
$$

where $x(n)=\left(x_{1}(n), \ldots, x_{d}(n)\right)^{T}, u(n)=\left(u_{1}(n), \ldots, u_{d}(n)\right)^{T}, p>0$; or a polynomial difference system

$$
\Delta x_{i}(n)=\sum_{j=1}^{\ell} a_{i j}(n) x_{1}^{q_{i j 1}}\left(n-\sigma_{j}(n)\right) \cdots x_{d}^{q_{i j d}}\left(n-\sigma_{j}(n)\right)+u_{i}(n)
$$

for $n \in \mathbb{Z}_{+}, i=1, \ldots, d$, where $q_{i j k} \in \mathbb{R}_{+}$for $i, k=1, \ldots, d$ and $j=1, \ldots, \ell$; or the scalar nonautonomous control system of the form

$$
\Delta x(n)=\frac{a(n) x^{p}\left(n-\sigma_{1}(n)\right)}{b(n)+x^{q}\left(n-\sigma_{2}(n)\right)}+u(n), \quad n \in \mathbb{Z}_{+},
$$

where $p, q>0$. In all the above cases assumption (2.2) holds under natural conditions with $\varphi(t)=t^{p}$ with some $p>0$.

We assume that the uncontrolled system, i.e., (2.1) with $u \equiv 0$ has unbounded solutions. Our goal is to find a positive diagonal matrix $D$ and a positive integer $k$ such that the delayed feedback law of the form

$$
u(n)=-D x(n-k)+r(n)
$$

guarantees that the closed-loop delayed system

$$
\begin{aligned}
\Delta x(n) & =g\left(n, x\left(n-\sigma_{1}(n)\right), \ldots, x\left(n-\sigma_{\ell}(n)\right)\right)-D x(n-k)+r(n), \quad n \in \mathbb{Z}_{+}, \\
y(n) & =C x(n), \quad n \in \mathbb{Z}_{+}, \\
x(n) & =\psi(n), \quad n \in\{-\tau,-\tau+1, \ldots, 0\}
\end{aligned}
$$

is BIBO stable. Here $r(n)$ is the reference input, $D=\operatorname{diag}\left(\lambda_{1}, \ldots, \lambda_{d}\right), \lambda_{i}>0$ for $i=1, \ldots, d, \psi \in S\left([-\tau, 0], \mathbb{R}^{d}\right)$ is the initial sequence associated to the equation where

$$
\tau:=\max \left\{\max _{1 \leq j \leq \ell}\left\|\sigma_{j}\right\|_{\infty}, k\right\},
$$

The assumed diagonal form of the feedback law (2.3) is one of the simplest possible choice. In its implementation it is important to know how large delay can be. In Theorem 2 and 3 we give sufficient conditions on how to select the feedback gain $D$ and the time delay $k$ to guarantee the boundedness of the solutions. Our conditions (see (4.1) and (4.13) below) show that the larger the delay the smaller gain can guarantee the boundedness of the solution.

Following [16], we introduce the next definition of BIBO stability. 
Definition 1. The closed loop system (2.4) is said to be BIBO stable if there exist positive constants $\theta_{1}$ and $\theta_{2}=\theta_{2}\left(\|\psi\|_{\tau}\right)$ such that every solution of the system (2.4) satisfies

$$
\|y(n)\| \leq \theta_{1}\|r\|_{\infty}+\theta_{2}, \quad n \in \mathbb{Z}_{+}
$$

for every reference input $r \in L^{\infty}\left(\mathbb{Z}_{+}, \mathbb{R}^{d}\right)$.

Later we need the notion of local BIBO stability (see similar definition in [1] for the continuous case).

Definition 2. The closed loop system (2.4) is said to be locally BIBO stable if there exist positive constants $\delta_{1}, \delta_{2}$ and $\theta$ satisfying

$$
\|y(n)\| \leq \theta, \quad n \in \mathbb{Z}_{+}
$$

provided that $\|\psi\|_{\tau}<\delta_{1}$ and $\|r\|_{\infty}<\delta_{2}$.

Our approach is the following. We associate the linear system

$$
\Delta z(n)=-D z(n-k), \quad n \in \mathbb{Z}_{+}
$$

with the constant delay $k \in \mathbb{N}$ and the initial condition

$$
z(n)=\psi(n), \quad-k \leq n \leq 0
$$

to (2.4). Then the state equation in (2.4) can be considered as the nonlinear perturbation of (2.6), and by the variation of constants formula (see, e.g., Lemma 4 in [4]) we get

$$
x(n)=z(n)+\sum_{j=0}^{n-1} W(n-j-1)\left[g\left(j, x\left(j-\sigma_{1}(j)\right), \ldots, x\left(j-\sigma_{\ell}(j)\right)\right)+r(j)\right]
$$

for $n \in \mathbb{Z}_{+}$, where $z(n)$ is the solution of (2.6)-(2.7) and $W$ is the fundamental matrix solution of (2.6), i.e., the solution of the IVP

$$
\begin{aligned}
\Delta W(n) & =-D W(n-k), \quad n \in \mathbb{Z}_{+}, \\
W(n) & = \begin{cases}\mathbf{0}, & -k \leq n \leq-1, \\
\mathrm{I}, & n=0 .\end{cases}
\end{aligned}
$$

Here $\mathrm{I} \in \mathbb{R}^{d \times d}$ is the identity matrix and $\mathbf{0} \in \mathbb{R}^{d \times d}$ is the zero matrix. Since $D$ is a diagonal matrix, it is easy to see that $W(n)$ is a diagonal matrix too for all $n \in \mathbb{Z}_{+}$.

We can rewrite the equation (2.8) as a VDE

$$
\begin{aligned}
x(n+1)= & z(n+1)+\sum_{j=0}^{n} W(n-j) g\left(j, x\left(j-\sigma_{1}(j)\right), \ldots, x\left(j-\sigma_{\ell}(j)\right)\right) \\
& +\sum_{j=0}^{n} W(n-j) r(j), \quad n \in \mathbb{Z}_{+},
\end{aligned}
$$


and so it is equivalent to

$$
x(n+1)=\sum_{j=0}^{n} f\left(n, j, x\left(j-\sigma_{1}(j)\right), \ldots, x\left(j-\sigma_{\ell}(j)\right)\right)+h(n), \quad n \in \mathbb{Z}_{+},
$$

where

$$
h(n):=z(n+1)+\sum_{j=0}^{n} W(n-j) r(j),
$$

and

$$
f\left(n, j, x^{(1)}, \ldots, x^{(\ell)}\right):=W(n-j) g\left(j, x^{(1)}, \ldots, x^{(\ell)}\right)
$$

for $0 \leq j \leq n, x^{(i)} \in \mathbb{R}^{d}, 1 \leq i \leq \ell$. The equation (2.10) is a nonlinear VDE with several delay functions.

\section{BOUNDEDNESS OF THE SOLUTIONS OF VDES WITH DELAYS}

In this section we give a general result for the boundedness of the solutions of nonlinear VDEs with multiple delays which is a natural extensions of the results presented in [3] for nonlinear VDEs without delays.

We consider the nonlinear VDE with several delays

$$
x(n+1)=\sum_{j=0}^{n} f\left(n, j, x\left(j-\sigma_{1}(j)\right), \ldots, x\left(j-\sigma_{\ell}(j)\right)\right)+h(n), \quad n \in \mathbb{Z}_{+},
$$

with the associated initial condition

$$
x(n)=\psi(n), \quad-\tau \leq n \leq 0,
$$

where $\tau$ is a positive integer constant. We assume the following conditions.

(B1) For any fixed $0 \leq j \leq n$ and $j, n \in \mathbb{Z}_{+}$

$$
f(n, j, \cdot, \ldots, \cdot): \underbrace{\mathbb{R}^{d} \times \ldots \times \mathbb{R}^{d}}_{\ell} \rightarrow \mathbb{R}^{d} .
$$

(B2) For any $0 \leq j \leq n$ and $1 \leq i \leq d$ there exists an $a_{i}(n, j) \in \mathbb{R}_{+}$such that

$$
\left|f_{i}\left(n, j, x^{(1)}, \ldots, x^{(\ell)}\right)\right| \leq a_{i}(n, j) \varphi\left(\max _{1 \leq m \leq \ell}\left\|x^{(m)}\right\|\right)
$$

holds for $x^{(1)}, \ldots, x^{(\ell)} \in \mathbb{R}^{d}$ with a monotone non-decreasing mapping $\varphi$ : $\mathbb{R}_{+} \rightarrow \mathbb{R}_{+}$, where $f=\left(f_{1}, \ldots, f_{d}\right)^{T}$.

(B3) $h(n)=\left(h_{1}(n), \ldots, h_{d}(n)\right)^{T} \in \mathbb{R}^{d}$ for $n \in \mathbb{Z}_{+}$.

(B4) $\sigma_{i}: \mathbb{Z}_{+} \rightarrow \mathbb{Z}_{+}$satisfies $\left|\sigma_{i}(n)\right| \leq \tau$ for $n \in \mathbb{Z}_{+}$and $i=1, \ldots, \ell$.

(B5) $\psi \in S\left([-\tau, 0], \mathbb{R}^{d}\right)$. 
Clearly, problem (3.1)-(3.2) has a unique solution under the above conditions. The next result formulates sufficient conditions implying the boundedness of the solutions.

Theorem 1. Let $\psi$ be fixed, (B1)-(B5) are satisfied and let $x(n ; \psi)$ be the solution of (3.1)-(3.2). Suppose there exist $N \in \mathbb{Z}_{+}, \mu \in \mathbb{R}_{+}$and $v \geq \mu$ such that for $i=$ $1, \ldots, d$

$$
\begin{gathered}
\sum_{j=0}^{N} a_{i}(N, j) \varphi(\mu)+\left|h_{i}(N)\right| \leq v, \\
\sum_{j=0}^{N} a_{i}(n, j) \varphi(\mu)+\sum_{j=N+1}^{n} a_{i}(n, j) \varphi(v)+\left|h_{i}(n)\right| \leq v, \quad n \geq N+1
\end{gathered}
$$

and

$$
\|x(n ; \psi)\| \leq \mu, \quad n \in\{-\tau, \ldots, N\} .
$$

Then the solution is bounded by $v$, i.e.

$$
\|x(n ; \psi)\| \leq v, \quad n \geq-\tau .
$$

Proof. Consider the solution $x(n)=x(n ; \psi), n \in \mathbb{Z}_{+}$of (3.1) with the initial condition (3.2), and let $\mu$ and $N$ be such that (3.6) holds. Then, by using (B2), (3.4), (3.6) and the monotonicity of $\varphi$, we have for $i=1, \ldots, d$

$$
\begin{aligned}
\left|x_{i}(N+1)\right| & \leq \sum_{j=0}^{N}\left|f_{i}\left(N, j, x\left(j-\sigma_{1}(j)\right), \ldots, x\left(j-\sigma_{\ell}(j)\right)\right)\right|+\left|h_{i}(N)\right| \\
& \leq \sum_{j=0}^{N} a_{i}(N, j) \varphi\left(\max _{-\tau \leq m \leq N}\|x(m)\|\right)+\left|h_{i}(N)\right| \\
& \leq \sum_{j=0}^{N} a_{i}(N, j) \varphi(\mu)+\left|h_{i}(N)\right| \\
& \leq v,
\end{aligned}
$$

Therefore $\|x(N+1)\| \leq v$, so (3.7) holds for $n=N+1$.

Now we show that (3.7) holds for any $n \geq N+1$. Assume, for the sake of contradiction, that there exists $n_{0} \geq N+1$ and $i_{0} \in\{1, \ldots, d\}$ such that

$$
\left|x_{i_{0}}\left(n_{0}+1\right)\right|=\left|x_{i_{0}}\left(n_{0}+1 ; \psi\right)\right|>v,
$$

and

$$
\left|x_{i}(n)\right|=\left|x_{i}(n ; \psi)\right| \leq v, \quad N+1 \leq n \leq n_{0}, \quad i=1, \ldots, d .
$$


Hence, from equation (3.1), we get

$$
\begin{aligned}
\left|x_{i_{0}}\left(n_{0}+1\right)\right| \leq & \sum_{j=0}^{N}\left|f_{i_{0}}\left(n_{0}, j, x\left(j-\sigma_{1}(j)\right), \ldots, x\left(j-\sigma_{\ell}(j)\right)\right)\right| \\
& +\sum_{j=N+1}^{n_{0}}\left|f_{i_{0}}\left(n_{0}, j, x\left(j-\sigma_{1}(j)\right), \ldots, x\left(j-\sigma_{\ell}(j)\right)\right)\right|+\left|h_{i_{0}}\left(n_{0}\right)\right| \\
\leq & \sum_{j=0}^{N} a_{i_{0}}\left(n_{0}, j\right) \varphi\left(\max _{-\tau \leq m \leq N}\|x(m)\|\right) \\
& +\sum_{j=N+1}^{n_{0}} a_{i_{0}}\left(n_{0}, j\right) \varphi\left(\max _{-\tau \leq m \leq j}\|x(m)\|\right)+\left|h_{i_{0}}\left(n_{0}\right)\right| .
\end{aligned}
$$

Since $\varphi$ is a monotone non-decreasing mapping, (3.5), (3.6) and (3.9) yield

$$
\left|x_{i_{0}}\left(n_{0}+1\right)\right| \leq \sum_{j=0}^{N} a_{i_{0}}\left(n_{0}, j\right) \varphi(\mu)+\sum_{j=N+1}^{n_{0}} a_{i_{0}}\left(n_{0}, j\right) \varphi(v)+\left|h_{i_{0}}\left(n_{0}\right)\right| \leq v .
$$

This contradicts to our hypothesis (3.8), so inequality (3.7) holds.

\section{MAIN RESULTS}

Our main goal in this section is to formulate sufficient conditions which grantee the BIBO stability of the closed loop system (2.4). We will assume that function $\varphi$ in (2.2) is a power function. Our first result is given for the case when $g$ in (2.2) has a sub-linear estimate, i.e., when $\varphi(t)=t^{p}$, with $0<p<1$ in (2.2).

Theorem 2. Let $g: \mathbb{R}^{d} \rightarrow \mathbb{R}^{d}$ be a function which satisfies inequality (2.2) with $\varphi(t)=t^{p}, 0<p<1, t \geq 0$. The feedback control system (2.4) with $D=$ $\operatorname{diag}\left(\lambda_{1}, \ldots, \lambda_{d}\right)$ and $k \in \mathbb{N}$ is BIBO stable if

$$
\|b\|_{\infty}:=\sup _{n \in \mathbb{Z}_{+}} b(n)<\infty \quad \text { and } \quad 0<\lambda_{i}<2 \cos \frac{k \pi}{2 k+1}, \quad i=1, \ldots, d
$$

hold.

Proof. Let $\psi=\left(\psi_{1}, \ldots, \psi_{d}\right)^{T} \in S\left([-\tau, 0], \mathbb{R}^{d}\right)$, and $z(n)=\left(z_{1}(n), \ldots, z_{d}(n)\right)^{T}$ be the solution of the IVP (2.6)-(2.7). Then, for $i=1, \ldots, d, z_{i}$ is the solution of the IVP

$$
\Delta z_{i}(n)=-\lambda_{i} z_{i}(n-k), \quad n \in \mathbb{Z}_{+}
$$

with initial condition

$$
z_{i}(n)=\psi_{i}(n), \quad-k \leq n \leq 0 .
$$


It is known (see, e.g., [4]) that condition (4.1) yields that there exists a positive constant $M$ and $\rho \in(0,1)$ such that

$$
\left|z_{i}(n)\right| \leq M\|\psi\|_{\tau} \rho^{n}, \quad n \in \mathbb{Z}_{+}, \quad i=1, \ldots, d,
$$

where $\|\psi\|_{\tau}:=\max _{-\tau \leq j \leq 0}\|\psi(j)\|$. Hence every solution of (4.2) tends to zero as $n \rightarrow \infty$, and

$$
\|z\|_{\infty}:=\sup _{n \in \mathbb{Z}_{+}}\|z(n)\| \leq M\|\psi\|_{\tau}<\infty .
$$

Let $W(n)=\operatorname{diag}\left(w_{1}(n), \ldots, w_{d}(n)\right)$ be the solution of (2.9). Relation (4.4) yields $\lim _{n \rightarrow \infty} w_{i}(n)=0$ for $i=1, \ldots, d$, and

$$
\eta:=\max _{0 \leq i \leq d} \sum_{n=0}^{\infty}\left|w_{i}(n)\right|<\infty .
$$

From (2.8), for all $n \in \mathbb{Z}_{+}$and $i=1, \ldots, d$, we have

$$
\begin{aligned}
x_{i}(n+1)= & z_{i}(n+1) \\
& +\sum_{j=0}^{n} w_{i}(n-j)\left[g_{i}\left(j, x\left(j-\sigma_{1}(j)\right), \ldots, x\left(j-\sigma_{\ell}(j)\right)\right)+r_{i}(j)\right],
\end{aligned}
$$

where $x(n)=\left(x_{1}(n), \ldots, x_{d}(n)\right)^{T}, g=\left(g_{1}, \ldots, g_{d}\right)^{T}$ and $r=\left(r_{1}, \ldots, r_{d}\right)^{T}$. Therefore (2.11) and (2.12) imply

$$
f_{i}\left(n, j, x^{(1)}, \ldots, x^{(\ell)}\right)=w_{i}(n-j) g_{i}\left(j, x^{(1)}, \ldots, x^{(\ell)}\right)
$$

and

$$
h_{i}(n)=z_{i}(n+1)+\sum_{j=0}^{n} w_{i}(n-j) r_{i}(j) .
$$

Hence, by (2.2),

$$
\begin{aligned}
\left|f_{i}\left(n, j, x^{(1)}, \ldots, x^{(\ell)}\right)\right| & \leq\left|w_{i}(n-j)\right|\left|g_{i}\left(j, x^{(1)}, \ldots, x^{(\ell)}\right)\right| \\
& \leq\left|w_{i}(n-j)\right| b(j) \varphi\left(\max _{-\tau \leq m \leq \ell}\left\|x^{(m)}\right\|\right),
\end{aligned}
$$

so the conditions (B1)-(B5) hold with $a_{i}(n, j):=\left|w_{i}(n-j)\right| b(j), 0 \leq j \leq n$.

By (4.1), (4.5), (4.6) and the definition of the infinity norm, we obtain

$$
\begin{aligned}
\gamma & :=\max _{1 \leq i \leq d} \sup _{n \in \mathbb{Z}_{+}}\left|h_{i}(n)\right| \\
& \leq \max _{1 \leq i \leq d} \sup _{n \in \mathbb{Z}_{+}}\left|z_{i}(n)\right|+\max _{1 \leq i \leq d} \sup _{n \in \mathbb{Z}_{+}} \sum_{j=0}^{n}\left|w_{i}(n-j)\right|\|r(j)\|
\end{aligned}
$$




$$
\begin{aligned}
& \leq \sup _{n \in \mathbb{Z}_{+}}\|z(n)\|+\|r\|_{\infty} \max _{1 \leq i \leq d} \sum_{j=0}^{\infty}\left|w_{i}(j)\right| \\
& =\|z\|_{\infty}+\eta\|r\|_{\infty}<\infty .
\end{aligned}
$$

By conditions (4.1) and (4.6) we get

$$
\begin{aligned}
\alpha: & =\max _{1 \leq i \leq d} \sup _{n \in \mathbb{Z}_{+}} \sum_{j=0}^{n} a_{i}(n, j) \\
& =\max _{1 \leq i \leq d} \sup _{n \in \mathbb{Z}_{+}} \sum_{j=0}^{n}\left|w_{i}(n-j)\right| b(j) \\
& \leq\|b\|_{\infty} \max _{1 \leq i \leq d} \sum_{j=0}^{\infty}\left|w_{i}(j)\right| \\
& =\eta\|b\|_{\infty}<\infty .
\end{aligned}
$$

Now we show that the inequalities (3.4) and (3.5) are satisfied with

$$
\varphi(t)=t^{p}, t \geq 0, \quad N=0, \quad \mu:=\|\psi\|_{\tau}:=\max _{-\tau \leq n \leq 0}\|x(n)\|
$$

and

$$
v:=\max \left(2\left(\eta\|b\|_{\infty}\|\psi\|_{\tau}^{p}+\|z\|_{\infty}+\eta\|r\|_{\infty}\right),\left(2 \eta\|b\|_{\infty}\right)^{\frac{1}{1-p}},\|\psi\|_{\tau}\right) .
$$

By using (4.9) and (4.10), it is clear that for $i=1, \ldots, d$

$$
a_{i}(0,0)\|\psi\|_{\tau}^{p}+\left|h_{i}(0)\right| \leq \eta\|b\|_{\infty}\|\psi\|_{\tau}^{p}+\|z\|_{\infty}+\eta\|r\|_{\infty} \leq v,
$$

therefore (3.4) holds with (4.11) and (4.12). We have $v \geq\left(2 \eta\|b\|_{\infty}\right)^{\frac{1}{1-p}}$, and so (4.10) and the definition of $\alpha$ yield

$$
v^{p-1} \alpha \leq \frac{\alpha}{2 \eta\|b\|_{\infty}} \leq \frac{1}{2}
$$

Similarly, using $v \geq 2\left(\eta\|b\|_{\infty}\|\psi\|_{\tau}^{p}+\|z\|_{\infty}+\eta\|r\|_{\infty}\right)$ and the inequalities (4.9) and (4.10), we obtain

$$
\frac{1}{v}\left(\alpha\|\psi\|_{\tau}^{p}+\gamma\right) \leq \frac{\alpha\|\psi\|_{\tau}^{p}+\gamma}{2\left(\eta\|b\|_{\infty}\|\psi\|_{\tau}^{p}+\|z\|_{\infty}+\eta\|r\|_{\infty}\right)} \leq \frac{1}{2}
$$

Thus

hence for all $n \geq 1$, we have for $i=1, \ldots, d$

$$
v^{p-1} \alpha+\frac{1}{v}\left(\alpha\|\psi\|_{\tau}^{p}+\gamma\right) \leq 1,
$$

$$
a_{i}(n, 0) \varphi\left(\|\psi\|_{\tau}\right)+\sum_{j=1}^{n} a_{i}(n, j) \varphi(v)+\left|h_{i}(n)\right| \leq \alpha\|\psi\|_{\tau}^{p}+\alpha v^{p}+\gamma \leq v,
$$


consequently, (3.6) holds with (4.11) and (4.12). Then all the conditions of Theorem 1 are satisfied, therefore the solution $x$ of the closed loop system (2.4) is bounded by $v$ for $n \geq-\tau$, i.e.,

$$
\begin{aligned}
\|x(n)\| & \leq v=\max \left(2\left(\eta\|b\|_{\infty}\|\psi\|_{\tau}^{p}+\|z\|_{\infty}+\eta\|r\|_{\infty}\right),\left(2 \eta\|b\|_{\infty}\right)^{\frac{1}{1-p}},\|\psi\|_{\tau}\right) \\
& \leq 2 \eta\|r\|_{\infty}+\max \left(2\left(\eta\|b\|_{\infty}\|\psi\|_{\tau}^{p}+\|z\|_{\infty}\right),\left(2 \eta\|b\|_{\infty}\right)^{\frac{1}{1-p}},\|\psi\|_{\tau}\right)
\end{aligned}
$$

for $n \geq-\tau$. Then

$$
\|y(n)\| \leq\|C\|\|x(n)\| \leq \theta_{1}\|r\|_{\infty}+\theta_{2}, \quad n \in \mathbb{Z}_{+},
$$

where $\theta_{1}:=2 \eta\|C\|$ and

$$
\theta_{2}:=\|C\| \max \left(2\left(\eta\|b\|_{\infty}\|\psi\|_{\tau}^{p}+\|z\|_{\infty}\right),\left(2 \eta\|b\|_{\infty}\right)^{\frac{1}{1-p}},\|\psi\|_{\tau}\right) .
$$

Hence, by Definition 1, the closed loop system (2.4) is BIBO stable.

It is easy to see that for $k=1$ the last inequality of (4.1) gives the upper bound $\lambda_{i}<1$, and as $k \rightarrow \infty$, the upper bound of $\lambda_{i}$ in condition (4.1) tends monotonically to 0 . Therefore large delay allows only small gain in the control law.

In the following theorem a sufficient condition is given for the BIBO stability in the case of a linear estimate of the function $g$.

Theorem 3. Let $g: \mathbb{R}^{d} \rightarrow \mathbb{R}^{d}$ be a continuous function which satisfies inequality (2.2) with $\varphi(t)=t, t \geq 0$. The closed loop system (2.4) with $D=\operatorname{diag}\left(\lambda_{1}, \ldots, \lambda_{d}\right)$ and $k \in \mathbb{N}$ is BIBO stable if

$$
\|b\|_{\infty}<\frac{1}{\eta} \quad \text { and } \quad 0<\lambda_{i}<2 \cos \frac{k \pi}{2 k+1}, \quad i=1, \ldots, d
$$

hold, where $\eta$ is defined by (4.6).

Proof. As in the proof of Theorem 2, we rewrite (2.4) in the form of (4.7), and define the functions $f_{i}, a_{i}$ and $h_{i}$ for $i=1, \ldots, d$. Then the conditions (B1)-(B5) are satisfied.

Next we show that the inequalities (3.4) and (3.5) are satisfied with

$$
\varphi(t)=t, \quad N=0, \quad \mu:=\|\psi\|_{\tau} \text { and } v:=\max \left(\frac{\eta\|r\|_{\infty}+M\|\psi\|_{\tau}}{1-\eta\|b\|_{\infty}},\|\psi\|_{\tau}\right) \text {, }
$$

where the positive constant $M$ is defined in (4.4), $\|\psi\|_{\tau}:=\sup _{-\tau \leq n \leq 0}\|x(n)\|$. Since $a_{i}(n, j):=w_{i}(n-j) b(j), 0 \leq j \leq n$, we have

$$
\sum_{j=0}^{n} a_{i}(n, j)=\sum_{j=0}^{n}\left|w_{i}(n-j)\right| b(j)
$$




$$
\begin{aligned}
& \leq \max _{1 \leq i \leq d} \sup _{n \in \mathbb{Z}_{+}} \sum_{j=0}^{n}\left|w_{i}(n-j)\right| b(j) \\
& \leq\|b\|_{\infty} \max _{1 \leq i \leq q} \sum_{n=0}^{\infty}\left|w_{i}(n)\right| \\
& =\eta\|b\|_{\infty} \\
& <1 .
\end{aligned}
$$

By (4.4), (4.8), (4.13), (4.14) and (4.16), we have for $n \in \mathbb{Z}_{+}, i=1, \ldots, d$

$$
\begin{aligned}
v \geq & \frac{\eta\|r\|_{\infty}+M\|\psi\|_{\tau}}{1-\eta\|b\|_{\infty}} \\
\geq & \frac{\|r\|_{\infty} \sum_{j=0}^{n}\left|w_{i}(n-j)\right|+\left|z_{i}(n+1)\right|}{1-\sum_{j=0}^{n}\left|w_{i}(n-j)\right| b(j)} \\
\geq & \frac{\sum_{j=0}^{n}\left|w_{i}(n-j)\right|\left|r_{i}(j)\right|+\left|z_{i}(n+1)\right|}{1-\sum_{j=0}^{n}\left|w_{i}(n-j)\right| b(j)} .
\end{aligned}
$$

Since $h_{i}(n)=\sum_{j=0}^{n} w_{i}(n-j) r(j)+z_{i}(n+1)$, it follows

$$
v \geq \frac{\left|h_{i}(n)\right|}{1-\sum_{j=0}^{n}\left|w_{i}(n-j)\right| b(j)}, \quad n \in \mathbb{Z}_{+} .
$$

Therefore

$$
v \sum_{j=0}^{n}\left|w_{i}(n-j)\right| b(j)+\left|h_{i}(n)\right| \leq v, \quad n \in \mathbb{Z}_{+}, \quad i=1, \ldots, d .
$$

Hence the above inequality and $v \geq\|\psi\|_{\tau}$ yield for $n=0$ and $i=1, \ldots, d$

$$
a_{i}(0,0)\|\psi\|_{\tau}+\left|h_{i}(0)\right| \leq v\left|w_{i}(0)\right| b(0)+\left|h_{i}(0)\right| \leq v,
$$

and so (3.4) is satisfied with (4.14). Similarly, for $n \in \mathbb{N}$ and $i=1, \ldots, d$

$$
v\left|w_{i}(n)\right| b(0)+v \sum_{j=1}^{n}\left|w_{i}(n-j)\right| b(j)+\left|h_{i}(n)\right| \leq v .
$$


Therefore

$$
a_{i}(n, 0)\|\psi\|_{\tau}+v \sum_{j=1}^{n} a_{i}(n, j)+\left|h_{i}(n)\right| \leq v, \quad n \in \mathbb{N}, \quad i=1, \ldots, d,
$$

consequently, (3.5) is satisfied with (4.14). Then all the conditions of Theorem 1 hold with with (4.14), therefore the solution $x$ of the closed loop system (2.4) is bounded by $v$, i.e.,

$$
\|x(n)\| \leq v, \quad n \in \mathbb{Z}_{+} .
$$

Hence

$$
\begin{aligned}
\|y(n)\| & \leq\|C\|\|x(n)\| \\
& \leq\|C\| v \\
& =\|C\| \max \left(\frac{\eta\|r\|_{\infty}+M\|\psi\|_{\tau}}{1-\eta\|b\|_{\infty}},\|\psi\|_{\tau}\right) \\
& \leq \theta_{1}\|r\|_{\infty}+\theta_{2},
\end{aligned}
$$

where

$$
\theta_{1}:=\frac{\|C\| \eta}{1-\eta\|b\|_{\infty}} \quad \text { and } \quad \theta_{2}:=\|C\| \max \left(\frac{M\|\psi\|_{\tau}}{1-\eta\|b\|_{\infty}},\|\psi\|_{\tau}\right) .
$$

Then, by Definition 1, the feedback control system (2.4) is BIBO stable.

Corollary 1. Let $g: \mathbb{R}_{+} \times \mathbb{R}^{d} \rightarrow \mathbb{R}^{d}$ be a continuous function which satisfies inequality (2.2) with $\varphi(t)=t, t \geq 0$. The closed loop system (2.4) with $D=$ $\operatorname{diag}\left(\lambda_{1}, \ldots, \lambda_{d}\right)$ and $k \in \mathbb{N}$ is BIBO stable if

$$
\|b\|_{\infty}<\lambda_{i} \leq \frac{k^{k}}{(k+1)^{k+1}}, \quad i=1, \ldots, d
$$

hold.

Proof. Under our condition (4.17) and from Lemma 4 in [4] we get that the fundamental solution $w_{i}$ of (4.2)-(4.3) is positive and

$$
\sum_{j=0}^{\infty} w_{i}(j)=\frac{1}{\lambda_{i}}, \quad i=1, \ldots, d .
$$

Therefore

$$
\eta=\max \left(\frac{1}{\lambda_{1}}, \ldots, \frac{1}{\lambda_{d}}\right)
$$

and hence $\eta\|b\|_{\infty}<1$. The proof is similar to the proof of Theorem 3 and it is omitted. 
In the next theorem it is shown that in the super-linear case there exist positive diagonal gain $D$ and positive delay $k$ such that the solutions of the closed loop system are bounded for small initial functions and small reference inputs, i.e., the system is locally BIBO stable.

Theorem 4. Let $g: \mathbb{R}^{d} \rightarrow \mathbb{R}^{d}$ be a continuous function which satisfies inequality (2.2) with $\varphi(t)=t^{p}, p>1, t \geq 0$. Then the solution $x$ of the feedback control system (2.4) is locally BIBO stable if (4.1) holds.

Proof. Suppose $\lambda_{1}, \ldots, \lambda_{d}$ are fixed satisfying (4.1), $D=\operatorname{diag}\left(\lambda_{1}, \ldots, \lambda_{d}\right)$, and let $z$ be the solution of the IVP (2.6)-(2.7), and $\eta$ be defined by (4.6). Let $\|\psi\|_{\tau} \leq \delta_{1}$ and $\|r\|_{\infty} \leq \delta_{2}$, where $\delta_{1}, \delta_{2}$ will be specified later. From (4.5) and (4.9) we have

$$
\|z\|_{\infty} \leq M\|\psi\|_{\tau} \leq M \delta_{1}
$$

and

$$
\gamma:=\max _{1 \leq i \leq d} \sup _{n \in \mathbb{Z}_{+}}\left|h_{i}(n)\right| \leq\|z\|_{\infty}+\eta\|r\|_{\infty} \leq M \delta_{1}+\eta \delta_{2}<\infty,
$$

and from (4.1) and (4.15) it follows

$$
\alpha:=\max _{1 \leq i \leq d} \sup _{n \in \mathbb{Z}_{+}} \sum_{j=0}^{n} a_{i}(n, j) \leq \eta\|b\|_{\infty}<\infty .
$$

Since $p>1$ and $\eta$ and $\|b\|_{\infty}$ are positive and finite, we select the positive constants $\delta_{1}$ and $\delta_{2}$ so that

$$
\alpha \delta_{1}^{p}+M \delta_{1}+\eta \delta_{2} \leq \frac{1}{2}\left(\frac{1}{2 \eta\|b\|_{\infty}}\right)^{\frac{1}{p-1}} \text { and } \quad 0<\delta_{1} \leq\left(\frac{1}{2 \eta\|b\|_{\infty}}\right)^{\frac{1}{p-1}}
$$

hold.

Next we show that the inequalities (3.4) and (3.5) are satisfied with

$$
\varphi(t)=t^{p}, \quad N=0, \quad \mu:=\|\psi\|_{\tau} \quad \text { and } \quad v:=\left(\frac{1}{2 \eta\|b\|_{\infty}}\right)^{\frac{1}{p-1}} .
$$

We note that the definitions of $\mu, \delta_{1}$ and the second part of (4.18) yield $\mu \leq v$. Using the definition of $v, p>1$ and (4.18) we get

$v \geq \frac{1}{2}\left(\frac{1}{2 \eta\|b\|_{\infty}}\right)^{\frac{1}{p-1}} \geq \alpha \delta_{1}^{p}+M \delta_{1}+\eta \delta_{2} \geq \alpha\|\psi\|_{\tau}^{p}+\gamma \geq a_{i}(0,0)\|\psi\|_{\tau}^{p}+\left|h_{i}(0)\right|$ for $i=1, \ldots, d$, hence the condition (3.4) holds with (4.19).

Similarly, the definition of $v, p>1$ and (4.18) yield

$$
v-\alpha v^{p} \geq v-\eta\|b\|_{\infty} v^{p}=\frac{1}{2}\left(\frac{1}{2 \eta\|b\|_{\infty}}\right)^{\frac{1}{p-1}} \geq \alpha \delta_{1}^{p}+M \delta_{1}+\eta \delta_{2} \geq \alpha\|\psi\|_{\tau}^{p}+\gamma .
$$


Then the definitions of $\alpha$ and $\gamma$ imply

$$
a_{i}(n, 0)\|\psi\|_{\tau}^{p}+\sum_{j=1}^{n} a_{i}(n, j) v^{p}+\left|h_{i}(n)\right| \leq \alpha\|\psi\|_{\tau}^{p}+\alpha v^{p}+\gamma \leq v, \quad n \in \mathbb{N},
$$

therefore the condition (3.5) holds with (4.19).

Therefore the conditions of Theorem 1 are satisfied with (4.19), so the solution of the closed loop system (2.4) is bounded by $v$, i.e.,

Hence

$$
\|x(n)\|<v=\left(\frac{1}{p \eta\|b\|_{\infty}}\right)^{\frac{1}{p-1}}, \quad n \in \mathbb{Z}_{+} .
$$

$$
\|y(n)\| \leq\|C\|\|x(n)\| \leq \theta, \quad n \in \mathbb{Z}_{+},
$$

where

$$
\theta:=\|C\|\left(\frac{1}{p \eta\|b\|_{\infty}}\right)^{\frac{1}{p-1}} .
$$

By Definition 2 the closed loop system (2.4) is locally BIBO stable.

\section{ACKNOWLEDGEMENT}

This research was partially supported by by the Hungarian National Foundation for Scientific Research Grant No. K120186.

\section{REFERENCES}

[1] E. Awwad, I. Győri, and F. Hartung, "BIBO stabilization of feedback control systems with time dependent delays," Appl. Math. Comput., vol. 219, pp. 3664-3676, 2012, doi: 10.1016/j.amc.2012.09.065.

[2] J. Cui, L. Xiong, and M. Ye, "BIBO stability of switched uncertain neutral control system," Int. J. Control Autom. Syst., vol. 4:8, pp. 913-923, 2010, doi: 10.1007/s12555-010-0427-x.

[3] I. Győri and E. Awwad, "On the boundedness of the solutions in nonlinear discrete Volterra difference equations," Adv. Difference Equ., vol. 2012, no. 2, 2012, doi: 10.1186/1687-1847-2012-2.

[4] I. Győri, G. Ladas, and P. N. Vlahos, "Global attractivity in a delay difference equations," Nonlinear Anal. Theory Meth. Appl., vol. 17, no. 5, pp. 473-479, 1991, doi: 10.1016/0362546X(91)90142-N.

[5] K. Hangos, J. Bokor, and G. Szederkényi, Analysis and Control of Nonlinear Process Systems. Springer, 2004. doi: 10.1007/b97665.

[6] S. Kotsios and N. Kalouptsidis, "BIBO stability criteria for a certain class of discrete nonlinear systems," Int. J. Contr., vol. 58, pp. 707-730, 1993, doi: 10.1080/00207179308923024.

[7] P. Li and S. Zhong, "BIBO stabilization of time-delayed system with nonlinear perturbation," Appl. Math. Comput., vol. 195, pp. 264-269, 2008, doi: 10.1016/j.amc.2007.04.081.

[8] P. Li and S. Zhong, "BIBO stabilization of piecewise switched linear systems with delays and nonlinear perturbations," Appl. Math. Comput., vol. 213, pp. 405-410, 2009, doi: 10.1016/j.amc.2009.03.029.

[9] P. Li, S. Zhong, and J. Z. Cui, "Delay-dependent robust BIBO stabilization of uncertain system via LMI approach," Chaos Solitons Fractals, vol. 40, pp. 1021-1028, 2009, doi: 10.1016/j.chaos.2007.08.059 
[10] Z. Li, H. Gao, and H. R. Karimi, "Stability analysis and $h_{\infty}$ controller synthesis of discretetime switched systems with time delay," Systems Control Lett., vol. 66, pp. 85-93, 2014, doi: 10.1016/j.sysconle.2013.12.010.

[11] M. Park, O. Kwon, and S. Choi, "Stability analysis of discrete-time switched systems with timevarying delays via a new summation inequality," Nonlinear Anal. Hybrid Syst., vol. 23, pp. 76-90, 2017, doi: 10.1016/j.nahs.2016.08.001.

[12] A. Seuret, F. Gouaisbaut, and E. Fridman, "Stability of discrete-time systems with time-varying delays via a novel summation inequality," IEEE Trans. Automat. Control, vol. 60, pp. 2740-2745, 2015, doi: 10.1109/TAC.2015.2398885.

[13] A. Trächtler, "On BIBO stability of systems with irrational transfer function," arXiv:1603.01059v1 [math.DS] 3 Mar 2016, 2016.

[14] B. Wang, J. Wang, X. Liu, and S. Zhong, "New results on BIBO stability analysis for a class of neutral delay systems," J. Franklin Inst., vol. 348, pp. 426-437, 2001, doi: 10.1016/j.jfranklin.2010.12.006.

[15] J. Wei, P. Shi, H. R. Karimi, and B. Wang, "BIBO stability analysis for delay switched systems with nonlinear perturbation,” Abstr. Appl. Anal., vol. Volume 2013, Article ID 738653, pp. 1-8, 2013, doi: 10.1155/2013/738653.

[16] H. Wu and K. Mizukami, "Robust stabilization of uncertain linear dynamical systems," Int. J. Syst. Sci., vol. 24, no. 2, pp. 265-276, 1993, doi: 10.1007/BF02192220.

[17] L. Xiong, S. Zhong, and M. Ye, "Delay-dependent BIBO stability analysis of switched uncertain neutral systems," Math. Comput. Modelling, vol. 53, pp. 1607-1620, 2011, doi: 10.1016/j.mcm.2010.12.026.

[18] D. Xu and S. Zhong, "Bibo stabilization of large-scale systems," Cont. Theory Appl., vol. 12, no. 6, pp. 758-763, 1995 .

[19] D. Xu and S. Zhong, "The BIBO stabilization of multivariable feedback systems," J. UEST China, vol. 24, no. 1, pp. 90-96, 1995.

Authors' addresses

Essam Awwad

Department of Mathematics, Faculty of Science, Benha University, Egypt

E-mail address: esam_mh@yahoo.com

István Győri

Department of Mathematics, University of Pannonia, Hungary

E-mail address: gyoriealmos.uni-panon.hu

Ferenc Hartung

Department of Mathematics, University of Pannonia, Hungary

E-mail address: hartung. ferenc@uni-pannon. hu 